\title{
¿Qué te lleva a moverte? Fenomenología de la práctica de actividad física por un largo plazo
}

\author{
What Motivates You to Exercise? Phenomenology of \\ Long-Term Physical Activity Practice
}

\author{
María Eugenia Tavernier Morga ${ }^{1}$ \\ (D) https://orcid.org/0000-0001-8234-4496 \\ Bernardo Turnbull Plaza² \\ https://orcid.org/0000-0001-9663-913X \\ Félix Guillén ${ }^{3}$ \\ https://orcid.org/0000-0002-1543-1351 \\ 1,2Departamento de Psicología, Universidad Iberoamericana, México \\ ${ }^{3}$ Departamento de Psicología y Sociología, Universidad de Las Palmas de Gran Canaria, España
}

Resumen. Objetivo. Conocer la experiencia de personas físicamente activas por un largo plazo desde tres etapas: inicio de la vida activa, motivación actual y afrontamiento a las barreras. Método. Se realiza un análisis fenomenológico a partir de entrevistas examinadas a profundidad. $N=11$ de personas adultas que han sido activas a un nivel suficiente por los últimos 10 años. Resultados. Surgieron cuatro categorías que describen: la vivencia autónoma, la decisión, el gusto adquirido desde la infancia y el hábito para regular emociones. A manera de conclusión, al conocer las características de la experiencia en la actividad física en personas activas, se confirmó aspectos sobre la motivación autodeterminada, lo que sugiere la presencia de elementos útiles en la intervención dirigida a la activación a largo plazo.

Palabras clave. Actividad física, motivación, vida activa, fenomenología, autonomía

Abstract. Objective. To know the experience of long-term active people to understand their relationship with physical practice in three stages: beginning of active life, current motivation, and facing barriers. Method. A phenomenological analysis was done on in-depth interviews. $N=11$ adults have been active at a sufficient level for, at least, the last 10 years. Results. Four categories were found: the autonomous experience, the decision making, the preference for an active life acquired since childhood, and the habit through which they regulate their emotions. As a conclusion, the characteristics of the experience of active people with physical activity confirmed aspects about self-determined motivation. Useful elements are suggested in the intervention aimed at long-term activation.

Keywords. Physical activity, motivation, active lifestyle, phenomenology, autonomy

\begin{abstract}
'María Eugenia Tavernier Morga. Departamento de Psicología, Universidad Iberoamericana, México. Dirección postal: Prolongación Paseo de la Reforma 880, Lomas de Santa Fe, Álvaro Obregón, 01219, Ciudad de México, México. E-mail: dra.eugeniatavernier@gmail.com ²Bernardo Turnbull Plaza. Departamento de Psicología, Universidad Iberoamericana, México. E-mail: bernardo.turnbull@ibero.mx ${ }^{3}$ Félix Guillén. Departamento de Psicología y Sociología, Universidad de Las Palmas de Gran Canaria, España. E-mail: felix. guillen@ulpgc.es
\end{abstract}

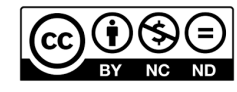

Esta obra está bajo una licencia de Creative Commons Reconocimiento-NoComercial-SinObraDerivada 4.0 Internacional. 


\section{Introducción}

El $42.4 \%$ de los adultos mexicanos residentes en zonas urbanas son activos en su tiempo libre, pero solamente la mitad de ellos realiza actividad física (AF) a un nivel suficiente para prevenir complicaciones de salud (Instituto Nacional de Estadística y Geografía [INEGI], 2018). Esta cifra es preocupante, ya que el riesgo de muerte por cualquier causa es más elevado en los adultos cuya AF es insuficiente que en aquellos que practican al menos 150 minutos de ejercicio físico moderado por semana (Organización Mundial de la Salud, 2014). Aunado a ello, la AF proporciona no solo beneficios físicos, sino que también, se asocia con una serie de variables que conforman una buena calidad de vida en las personas (Guillén et al., 1997).

Las implicaciones de la insuficiente AF en la población mexicana son alarmantes, ya que se ha llegado al grado de declararse una emergencia epidemiológica por obesidad y diabetes mellitus en el país (Secretaría de Salud, 2016), las cuales son dos de las consecuencias de la inactividad física. Por esto, potenciar la práctica de AF debe ser una tarea prioritaria no solo para la prevención, sino también para el combate a estos padecimientos.

\section{Motivación para la actividad física}

Uno de los aspectos principales en el estudio de la AF desde una perspectiva psicológica es la motivación (Roychowdhury, 2018). La teoría de la autodeterminación (TAD; Ryan \& Deci, 2000) es una macro-teoría de la motivación humana y de la personalidad en contextos sociales. Esta concibe al ser humano con una tendencia natural hacia el crecimiento y hacia la integración, si los factores ambientales lo favorecen. Sostiene además que las personas tenemos tres necesidades psicológicas innatas: competencia, autonomía y relación. Los elementos del contexto social y ambiental van a ampliar o limitar la satisfacción de tales necesidades (Deci \& Ryan, 2008).

Ahora bien, la autonomía puede explorarse desde tres sentidos (Ryan et al., 2021), los cuales son el compromiso conductual, su impacto en la sostenibilidad de relaciones interpersonales y su relación con los contextos socioculturales y económicos. Es de interés para el estudio de la permanencia en la AF el compromiso conductual, ya que representa a la autonomía como un estado en el cual la conducta propia es auto-organizada y voluntaria. Al respecto, Sansone y Tang (2021) declaran que uno de los retos actuales es determinar si la autonomía es causa o resultado del compromiso con la actividad que se realiza, y si se requiere de mayor exploración acerca de lo que realmente hacen las personas que se enganchan en un determinado quehacer.

La TAD aborda también los tipos de objetivos de vida intrínsecos y extrínsecos que mueven a las personas hacia el rendimiento y la salud psicológica (Deci \& Ryan, 2008). En el campo de la $A F$, se ha encontrado que los contenidos de metas de tipo intrínseco son el manejo de la salud, el desarrollo de habilidades y la afiliación social, mientras que los extrínsecos son el reconocimiento social y la imagen. Los objetivos intrínsecos se correlacionan positivamente con la regulación autónoma, pero no necesariamente con la satisfacción de la necesidad de autonomía (Sebire et al., 2008). Recientemente, Valenzuela et al. (2021) han estudiado la relación entre los motivos de la AF y la satisfacción de necesidades psicológicas básicas en estudiantes universitarios. En su estudio, encontraron cuatro grupos motivacionales (el intrínseco, el extrínseco, el de alta puntuación en todos los motivos y el de baja puntuación en todos los motivos). Descubrieron además que, aunque algunos motivos intrínsecos sí se relacionan con la satisfacción de la necesidad de autonomía, esto no implica que a mayor motivación intrínseca exista mayor satisfacción 
de tal necesidad. Asimismo, los motivos extrínsecos contribuyeron a la satisfacción de autonomía. Adicionalmente, Ntoumanis et al. (2014) reportaron que los motivos autónomos predicen la persistencia en la AF cuando las personas se enfrentan a objetivos de creciente intensidad. Respecto al mantenimiento de la práctica de AF a largo plazo, en una revisión sistemática, se ha documentado la relevancia que tiene un entorno que promueve la satisfacción de las necesidades psicológicas básicas y que fomenta una regulación autónoma en la adherencia a la conducta activa (Rodrigues et al., 2018).

En suma, la TAD ha mostrado importante evidencia de su valor tanto en el contexto de la AF (Teixeira et al., 2012) como en el de las conductas relacionadas a la salud ( $\mathrm{Ng}$ et al., 2012). Esta teoría representa un enfoque prometedor en el área de innovaciones en salud dirigidas al cambio conductual para un estilo de vida saludable (Migliorini et al., 2019).

\section{Motivos para práctica de actividad física}

Los principales motivos que refieren los mexicanos y las mexicanas para realizar AF son salud, diversión y el aspecto físico (INEGI, 2018). Por otro lado, los motivos recurrentes para la inactividad física, de acuerdo con el Instituto Nacional de Salud Pública ([INSP], 2016), son la falta de tiempo, el cansancio por el trabajo, los problemas de salud, la pereza y la falta de dinero. Se señalan además como obstáculos la escasez de espacios adecuados y seguros, la ausencia de motivación, la preferencia por actividades sedentarias, la falta de AF en la familia, los problemas de salud y el desagrado por hacer ejercicio.

Ashton et al. (2015) describieron los motivadores para la participación en AF en hombres jóvenes de Australia: la apariencia física, la inclusión social, la salud física y mental y la mejora en el deporte o fitness. Este estudio también reportó como barreras: un estilo de vida ocupado, problemas logísticos como el alto costo, los sentimientos de inferioridad y algunos factores sociales como la crianza. En Turquía, por otro lado, los hombres muestran más motivos en la apariencia física y en la competición que las mujeres (Sarol \& Çimen, 2017). Estos resultados coinciden con lo hallado en España en un estudio comparativo por género (Leyton et al., 2018) donde se mostró que los varones se destacaron por tener motivos competitivos.

Por otra parte, existe evidencia de que los motivos intrínsecos se asocian con la AF constante en el tiempo libre, tal como lo demostraron Aaltonen et al. (2014) al comparar entre las poblaciones finlandesas activas y las inactivas. Los autores reportaron que la maestría deportiva, la aptitud física, los aspectos sociales relacionados al deporte, el estado psicológico, el disfrute, el deseo de ser mejor que otros y la apariencia física presentan diferencias estadísticamente significativas entre ambos grupos, siendo estos factores más intensos entre el grupo de personas regularmente activas.

A través de un estudio más específico sobre la comparación de motivos, Ball et al. (2014) buscaron diferencias entre los practicantes de deporte, de ejercicio y de AF recreativa. Los autores encontraron que son semejantes en cuanto a motivación quienes hacen ejercicio y quienes realizan AF recreativa para obtener fuerza y resistencia y controlar su peso corporal y el estrés. Por contraste, los que practican deportes se mueven más por competición y disfrute. Los resultados de este trabajo además contrastan con el estudio de Aaltonen et al. (2014), ya que destacan que son los motivos extrínsecos los que se relacionan con los tipos de práctica del ejercicio y la AF recreativa. A esta inconsistencia en los resultados de las investigaciones se le suma lo encontrado por Stelzer et al. (2018), que concluyen que la motivación para participar en la AF no está suficientemente relacionada con los altos niveles de esa conducta, lo que abre paso a la interrogante para genere una investigación que contribuya a esclarecer ese hallazgo. 
Por otra parte, en un estudio comparativo, se encontró que la población adolescente mexicana, en contraste con la española y costarricense, resulta ser la más inactiva y desmotivada (Ruiz-Juan et al., 2017). En Brasil, Guillén et al. (2005) encontraron que los motivos que llevan a los jóvenes a la práctica deportiva presentan ciertas diferencias según su edad. Estas razones aparecen en algunos aspectos concretos y en los factores que los conforman. En cualquier caso, los motivos señalados son semejantes a los encontrados en otros estudios realizados en países como España y EE. UU. Circunscribiéndose a jóvenes deportistas también de México, los motivos frecuentemente citados son para estar en forma, para estar fuerte y saludable, por gusto y para mejorar sus habilidades (RodríguezMartínez \& Guillén, 2017).

En resumen, las encuestas nacionales reportan los motivos de una población que engloba activos e inactivos; los estudios con grupos específicos se han enfocado en deportistas de rendimiento o en edades juveniles, y los estudios internacionales, si bien brindan una buena orientación, son ajenos a la situación vivida en México. Por tanto, existe falta de información, en nuestro contexto, de la población adulta regularmente activa que no está necesariamente involucrada en un tipo de AF competitiva y de cuya constancia en el ejercicio podamos aprender. Es decir, es necesario escuchar la voz de ciudadanos con las comunes actividades laborales y domésticas referidas en encuestas como barreras para la AF, pero que, a pesar de ello, tienen años siendo activos. Potenciar este tipo de conducta sería el objetivo deseado para mejorar los indicadores de salud en México.

Con base en la estrecha relación existente entre las conductas de promoción de salud y los conceptos de salud que subyacen a las mismas (Levesque \& Li, 2014), se considera importante conocer sobre el concepto de AF en el grupo de personas antes definidas, debido a que algunas investigaciones han evidenciado que existe gran variabilidad en la conceptualización de la AF por la cultura (Alvarado et al., 2015), el género (LozadaTequeanes et al., 2015) o la enfermedad (López-Amador \& Ocampo-Barrio, 2007). Asimismo, poco se ha estudiado sobre ello en la población activa dentro del contexto social descrito anteriormente. Algunos investigadores del área de la motivación en la AF han recomendado, luego de sus hallazgos, realizar investigación en profundidad (Rodríguez-Martínez \& Guillén, 2017) o de tipo cualitativa (Sarol \& Çimen, 2017) a fin de ahondar en este complejo fenómeno. El abordaje cualitativo permite una comprensión del tema con una mayor validez ecológica, en otras palabras, atiende a la vivencia de las personas en su vida cotidiana con todas las vicisitudes que esto implica. Se espera que la información obtenida sea apegada al funcionamiento real de estas con el fin de extraer lo aplicable a una mayor extensión de personas.

Por lo anteriormente expuesto, queda la tarea de saber ¿cuál es la experiencia de quienes, siendo minoría, se han mantenido activos por un largo plazo y han superado las barreras para la AF que declara la mayoría de la población mexicana? Más allá de los números, en este estudio, se busca entender cómo ser activo es vivido por las personas que se desenvuelven en un medio en el que lo común es no serlo. Concretamente, el objetivo general es abstraer la esencia de su relación con la práctica físico-deportiva en personas perseverantes en la AF. Las dimensiones determinadas para esta abstracción son: el inicio de la vida activa, la motivación actual y el afrontamiento a las barreras.

\section{Método}

Esta es una investigación de corte transversal, de tipo cualitativa. Para el análisis de la información, se eligió el método fenomenológico porque se enfoca en la búsqueda del 
significado de la experiencia (Creswell, 2009), la cual promete un camino de respuesta que lleva a conocer la vivencia de las personas activas en un contexto de alta frecuencia de problemas de salud relacionados con la inactividad física.

\section{Participantes del estudio}

Se alcanzó la saturación teórica con la participación de 11 personas, de las cuales 6 hombres $(M=37.1$ años, rango de edad 26-45 años) y 5 mujeres ( $M=39.6$ años, rango de edad 35-45 años) son habitantes de la ciudad de Cancún, Quintana Roo. De esta población, 7 vivían en matrimonio, 4 en soltería y 8 tenían hijos. Respecto a su constitución física, los hombres tuvieron un índice de masa corporal medio de 25.44 ( \pm 1.07$)$ mientras que el de las mujeres fue de 22.84 ( \pm 1.35 ). Asimismo, de acuerdo con su tipo de AF, 7 asistían regularmente a un gimnasio, 3 pertenecían a equipos deportivos (fútbol bandera, fútbol y basquetbol respectivamente) y uno practicaba carreras al aire libre. Ningún participante tenía relación personal, laboral o académica con los investigadores.

Para el reclutamiento de los participantes se realizó un muestreo intencional cuyo principal criterio de participación fue la práctica regular de AF (mínimo tres veces por semana), por lo menos, en los últimos diez años. Se tuvo especial atención en observar que se tratara de personas adultas sanas. Se definieron como criterios de exclusión: tener una ocupación laboral relacionada con el ámbito de la activación física o deporte y ser estudiante. Se orientó la búsqueda en centros de entrenamiento públicos y privados donde inicialmente se contactó a entrenadores para obtener información sobre potenciales participantes. Una vez identificados, se les planteó de forma individual participar en la investigación mediante una entrevista cara a cara, en la que ellos tuvieron la posibilidad de elegir el horario y lugar a fin de promover el clima de confianza necesario para obtener los datos. Una entrevista fue descartada del análisis debido a que la persona refirió haber estado en tratamiento por el padecimiento de bulimia.

La población participante brindó su consentimiento informado en apego a los lineamientos de la Declaración de Helsinki (World Medical Association, 2013), y ninguno recibió retribución alguna. El periodo en el que se llevaron a cabo las entrevistas fue de septiembre a diciembre del 2017.

Recolección de datos

Los datos fueron generados a partir de entrevistas en profundidad, las cuales tienen como ventaja la posibilidad de ahondar en ciertos temas, pero a la vez pueden ser muy amplias, ya que incorporan contenidos ajenos a la investigación. Por tanto, se definieron tres ejes sobre los cuales centrar la atención que están en línea con las dimensiones del objetivo general: el inicio de la vida activa, la motivación actual y el afrontamiento de las barreras a la AF. Todas las entrevistas fueron conducidas por la primera autora de este estudio, quien posee experiencia en entrevista psicológica. Cada entrevista se realizó en una sola sesión con duración media de 28 minutos. Estas fueron almacenadas en una grabadora marca Sony modelo ICD-PX333 y posteriormente fueron transcritas de manera fiel usando un pseudónimo para preservar la confidencialidad de los participantes.

\section{Análisis}

El procedimiento de codificación fue realizado en el software Atlas.ti, versión 8.3.17 (2018), donde se cargaron previamente las transcripciones como documentos de trabajo, y se procedió con un estilo de codificación abierto, es decir, sin categorías previamente establecidas. 
Después de lecturas repetidas, se identificaron citas significativas que describían las experiencias de los participantes y se generaron códigos para ellas. Una vez alcanzados los criterios de saturación teórica y de código (Hennink et al., 2017), se agruparon en cuatro áreas temáticas.

Las acciones para conferir rigor al estudio están enmarcadas en la búsqueda de integridad metodológica (Levitt, 2020), la cual es consistente con un construccionismo epistemológico y una visión ontológica relativista, que son la fundamentación del método fenomenológico aquí utilizado (Smith \& McGannon, 2018). Concretamente, se realizaron las siguientes estrategias: todas las entrevistas fueron conducidas a profundidad por una entrevistadora con experiencia en estas y de profesión psicóloga; en la categorización, se respetaron los temas expresados por las personas, sin imponer modelos teóricos a priori; se realizó la búsqueda de la máxima variación en el muestreo; y se declaró que los datos construidos en la interacción entrevistador-participante no pueden ser replicados; sin embargo, se recurrió a la técnica de amigos críticos (Smith \& McGannon, 2018) para estimular la reflexión sobre los discursos y el proceso de investigación.

\section{Resultados}

A partir del análisis, emergieron cuatro temas centrales: la realización de AF como un hábito esencial, la motivación autónoma, los motivos y la difusión. A continuación, se desarrollan todos estos temas junto con sus respectivos subtemas específicos:

Tema 1. Hacer actividad física es un hábito esencial

Los participantes describieron su AF como parte de una rutina diaria, dentro de la cual esta actividad tiene un carácter prioritario. Los participantes calificaron esta práctica regular en términos de costumbre, hábito o un vicio de los buenos. La declaración del participante ejemplifica esta descripción:

Y fue ahí cuando se me hizo un hábito yo creo, porque ya es un hábito ir al gimnasio todos los días. Ya lo veo como parte de mi vida diaria pues. Independientemente de que esté muy cansado o de que sean vacaciones. Incluso, cuando salgo de la ciudad, siempre procuro ver que en el hotel en el que me voy a quedar tenga algún área deportiva para que no descuide yo esa parte (Juan).

A partir de esta concepción general de la AF como parte de su rutina diaria, se identificaron dos propiedades: "Ios pretextos no existen" y "ejercitarse es fácil".

Los pretextos no existen. Una propiedad en esta categoría es que los participantes no perciben barreras para la ejercitación. Tienen la idea de que hacer ejercicio es una cuestión personal sobre la que no existen asuntos externos que puedan limitar o impedir dicha práctica. Si no se tiene el dinero para pagar un club de entrenamiento, eligen actividades en sitios públicos o en el hogar; si el tiempo libre es escaso, buscan despertarse más temprano, priorizando siempre el entrenamiento, pues, de igual manera, para ejercitarse no hace falta un espacio determinado. Para el grupo entrevistado, el movimiento radica en el cuerpo y no depende de un equipamiento o instalación especializada. Sus ejemplos son parques y, con suma frecuencia, el propio hogar. Algunos refieren al gimnasio más cercano, no al más equipado. Por ejemplo, Lesli contó que "subía y bajaba escaleras, hacía sentadillas y diferentes, pero me hacía mis 40 minutos de $A F$, porque no tenía tiempo de ir al gimnasio". 
Ejercitarse es fácil. La mayoría de los entrevistados coincidieron en la percepción de que hacer ejercicio es fácil, apreciación a la que llegan en contraste con lo que perciben de otros. Es decir, expresan que son conscientes de que, para otras personas, hacer ejercicio puede ser un tormento, pero nunca han sentido esto. Esta percepción la atribuyen al gusto que tienen por la práctica misma de la AF. Sin embargo, uno de los participantes, cuyo objetivo es el aumento de masa muscular, dijo que, en ese periodo en específico, ejercitarse le demandaba mucho esfuerzo y le resultaba muy cansado. El grupo participantes percibe además que quienes los rodean tienen una percepción de ellos como obsesionados o muy exigentes consigo mismos; sin embargo, esto no es algo que los detenga o los haga reconsiderar su regularidad en la AF.

\section{Tema 2. Motivación autónoma}

Se agruparon en esta categoría las propiedades de decisión, automotivación y autonomía, las cuales conforman un espectro de conductas y experiencias buscadas bajo la propia voluntad y la reafirmación de sí mismos de manera continua.

Decisión. Los participantes refieren que la práctica regular de AF la iniciaron a partir de una decisión propia. Una participante lo expresó así: "yo digo que es decisión de uno y que te gusta" (Zory).

Automotivarse. La población entrevistada narra un tipo de auto-diálogo para motivarse, el cual se compone de elementos de amor propio. Con frecuencia se refieren al tiempo para la AF como un tiempo que se regalan para sí. Como ejemplo, una de las mujeres dijo "es que, si tú no lo haces, nadie te va a venir a decir ¡hey!" (Lesli).

Autonomía. La decisión y la práctica de AF son completamente personales e independientes, es decir, son autónomas. Con frecuencia, las personas entrevistadas afirmaron no necesitar a alguien que los acompañe a realizar su ejercitación. Por el contrario, detectan cuando otras personas tratan de influenciar negativamente su práctica y se alejan de estas. Al cuestionarle a una participante sobre si hay algo que le dificulte su práctica, ella explicó que "es personal (...) no hay nada ajeno o fuera de que me haga falta para mantenerme así" (Liliana).

\section{Tema 3. Motivos para realizar actividad física}

Las razones citadas por las que hacen ejercicio físico (EF) se constituyeron en las siguientes propiedades: verse bien, gusto al practicarlo, beneficios a la salud mental y preservación de la salud física.

Verse bien. Uno de los motivos más enfatizados fue verse bien, ya que es tanto un deseo como un resultado del ejercicio. En términos de los participantes, al observar cambios favorables en su cuerpo se ven motivados a preservarlos. Aunque hombres y mujeres comparten este motivo, el mantener un peso/talla que sea estéticamente aceptable tiene un significado distinto entre ambos. Por un lado, ellas evitan ganar peso; por otro, ellos reportaron que ganar peso (musculatura) es parte de verse bien. Uno de los participantes varones lo describió de esta forma: "cuando yo entré a la preparatoria no me gustaba ser tan delgado (...) entonces fue que decidí meterme al gimnasio" (Juan). En contraste, una entrevistada agregó: "yo hago ejercicio porque me gusta ejercitar el músculo, no es estar flaca escuálida, yo digo flaca sin chiste, tener figura y más que nada por eso siempre he practicado el ejercicio" (Lesli). 
Por el gusto de practicarlo. El disfrute de la actividad fue una razón muy repetida. Sin mayor detalle, los entrevistados dicen que desde siempre les ha gustado hacer algún tipo de AF. Cecilia lo manifestó así: "pues me gusta, me agrada el moverme, el bailar, pues con esto que hago zumba, el bailar, el aprender a bailar, pues también me gusta".

Por sus beneficios a la salud mental. En este sentido, los participantes valoran la reducción del estrés que sienten luego del EF, así como una mejoría en el humor y en la actitud para afrontar las actividades cotidianas. También, describieron que, mientras realizan EF, evitan tener pensamientos asociados con el estrés, la ansiedad o las cuestiones negativas: "es que de verdad no hay mejor terapia para desestresarme que hacer ejercicio. Créeme que es lo mejor", expresó Lesli.

Para preservar la salud física. En esta categoría, esta población ejercitada dijo que sentirse en un buen estado de salud es importante para mantenerse activo. Es una necesidad que su cuerpo demanda para darle el mantenimiento adecuado: "por disciplina sería porque, obviamente, siempre nos han comentado que hacer ejercicio nos mantiene sanos físicamente y por ende nos da una salud adecuada, ¿no?", así lo representó Jil.

\section{Tema 4. Difusión de la actividad física}

Esta categoría implica la transmisión de información y actitudes propias sobre la AF, la cual se da tanto dentro como fuera del sistema familiar y en distintas direcciones. En este sentido, se obtuvieron las propiedades del ámbito familiar y la del contagio al círculo social, los cuales corresponden a los dos espacios de difusión referidos.

Ámbito familiar. La experiencia con la familia abarca el grupo familiar del que se proviene y el que se forma. Respecto al primer punto, los padres y las madres ejercieron una influencia importante durante la infancia de los participantes, en el sentido de fomentar la AF. Esta influencia pudo haber sido directa o indirecta. En el primer caso, los padres y las madres fueron ejemplo de la conducta activa:

Realmente, desde pequeños hemos estado creciendo a la par de las actividades físicas, porque hasta para ir al campo o ir a algún lugar de diversión, siempre era estar aprendiendo algo sobre algún deporte o estarlo practicando simplemente por diversión o por disciplina (Jil).

Cuando la influencia es indirecta, se refiere a que los padres y las madres no practicaron deporte, lo que les ocasionó un deterioro en la salud que la población entrevistada mencionó como lección que desean evitar. En este sentido, la persona comentó:

O sea, es todo lo contrario porque pues mis papás son gorditos. Desde chiquita me metieron en el deporte, pero ellos no hacían, o sea siempre yo dije que no quiero estar gordita para no tener los problemas de salud que tiene mi mamá o dolores de rodilla, así como mi papá (Paty).

En relación con la familia que han conformado, las personas que tienen hijos e hijas refieren que el trasmitirles la AF como un hábito es básico en el proceso de crianza. Otorgan gran importancia el pasar tiempo de ejercitación con sus descendientes.

El contagio al círculo social. En esta propiedad, la persona transmite, de alguna manera, su hábito por el ejercicio hacia sus compañeros de trabajo y amistades. Distinto a la forma en la que se hace con los hijos y las hijas, en la que se tiene como propósito explícito el dotarlos de prácticas saludables, el contagio se da por el ejemplo que se representa y por la imagen física lograda: 
Por lo general, las personas que están a mí alrededor se contagian de esa motivación. (...) por ejemplo, he tenido novias desde que inicie la universidad y mi novia actual y mis dos exnovias por fuerza se tuvieron que meter al gimnasio, no sé si para acompañarme o para pasar más tiempo conmigo. Pero por ejemplo a mi exnovia ya le quedó como un hábito y ya no andamos y todo, y ella sigue haciendo ejercicio y procura cuidarse en su alimentación (Juan).

\section{Discusión}

En respuesta a la pregunta planteada acerca de la experiencia de las personas que han mantenido una práctica físico-deportiva a lo largo de su vida, se encontró que la AF es vivida como una actividad predominantemente autónoma, producto de una decisión y un gusto adquirido desde la infancia. Esta se establece en la cotidianeidad de la persona como un hábito por medio del cual pueden regular favorablemente sus emociones, lo que, a su vez, les permite perseverarse en dicha práctica.

Hacer AF es un hábito esencial, y como primer aspecto surgido del análisis, se hace referencia al carácter repetitivo de esta práctica. Sin embargo, a esta se la califica de esencial, ya que transciende el concepto de puro de hábito, el cual se define como un proceso por el cual una señal dispara automáticamente un impulso a actuar basado en una asociación señal-conducta (Gardner, 2015) aprendida a través de repetidas ejecuciones (Gardner et al., 2019). Para los participantes, la EF tiene un carácter primordial en su rutina diaria, pues la perciben con agrado y crean soluciones ante cualquier posible complicación para su práctica. Aluden también al sentido de normalidad con el que han incorporado el concepto de AF en su vida cotidiana, y se constata en la manera en que lo conocido teóricamente como barreras para la práctica de EF no existe para ellos.

La motivación autónoma, como otra de las temáticas aquí surgida, es consistente con lo reportado en cuanto al efecto que tiene sobre la adherencia a largo plazo de la AF recreativa vigorosa (Hartmann et al., 2015; Rodrigues et al., 2018). Los componentes de esta motivación autónoma (decisión, autonomía y automotivación) confirman el constructo de motivación autodeterminada (Ryan \& Deci, 2000).

Una de las características de la forma de motivación descrita por los entrevistados fue codificada como un auto-diálogo. Llama la atención como al menos en la co-construcción que implica la entrevista, la población entrevistada narra conversaciones consigo mismos con una intención clara de darse el ánimo suficiente para perseverar en el ejercicio o deporte en los momentos en los que se está en riesgo de abandonarlo. De acuerdo con la clasificación propuesta por Latinjak et al. (2018), podríamos decir que se trata de un auto-habla estratégico motivacional. Valdría la pena profundizar en este aspecto cognitivo en futuras investigaciones que se centren en las actividades físicas para la salud y la recreación, ya que esto ha sido mayormente abordado para el rendimiento deportivo. Con los resultados aquí presentados, se observa que esta estrategia motivacional sucede en momentos cruciales para evitar el abandono de la EF en la población.

Respecto al tipo de motivos reportados, llama la atención la combinación de los motivos extrínsecos (verse bien, el más enfáticamente señalado) con los intrínsecos (gusto, salud mental y física). Un hallazgo similar fue presentado por Sicilia et al. (2014), estudio en el que una población española asidua practicante de AF señaló motivos autodeterminados y no autodeterminados en casi la misma intensidad. También, en un análisis cualitativo en corredores recreativos (Goodsell et al., 2013), los más consistentes en la actividad fueron quienes refirieron motivos tanto sociales como personales. En suma, los motivos para 
un hábito de permanencia en la AF son mucho más complejos que los que se presentan en la iniciación; se requiere no solo recompensas, sino también control personal y comportamiento repetitivo. En general, es un área en la que aún queda mucho por explorar (Lee \& Yoon, 2019). Estos hallazgos en conjunto sugieren que la sinergia de una red de motivos intrínsecos y extrínsecos favorece el mantenimiento en la AF en un largo plazo. Además, apoyan la reciente perspectiva de que la autonomía también puede verse beneficiada por los motivos extrínsecos (Ryan et al., 2021), lo cuales pueden originarse al interior de las personas y contribuir al bienestar al sintonizarse con sus propios valores (Sansone \& Tang, 2021).

Tres de los motivos encontrados son similares a los que arrojó la Encuesta Nacional de Salud y Nutrición (INSP, 2016), los cuales son la salud, la diversión (equiparable al placer) y el atractivo físico. Sin embargo, la salud mental como motivo se distinguió en la muestra y, junto con el placer por la AF, podría ser la clave para la permanencia a largo plazo. Esto debido a que, en sí mismos, estos motivos implican un refuerzo para la AF. La diferencia en los resultados podría explicarse porque en la encuesta nacional se contemplan personas con suficiente e insuficiente nivel de $A F$, $y$, en el presente trabajo, la muestra la comprende una población suficientemente activa. En un estudio comparativo entre mujeres practicantes y no practicantes de actividad físico-deportiva en España (Moreno-Murcia et al., 2016), se encontró que los motivos predominantes para las primeras fueron la salud y el desarrollo de la habilidad. Estos resultados son bastante consistentes con lo aquí reportado al considerar el paralelismo entre el gusto de practicar la AF y el desarrollo de la habilidad, pues ambos refieren a cuestiones específicas de la tarea deportiva. En otro estudio realizado en Madrid (Rodríguez-Romo et al., 2018), se obtuvo que, a mayor edad, aumentan los motivos de salud y disminuyen los de competencia, lo cual da sentido al hecho de que la competencia no fue mencionada por los participantes adultos que ya han superado la etapa universitaria, la cual implica muchas veces competencia en la práctica deportiva.

Es notable, entre los hallazgos, el hecho de que las personas confieren a la AF un sentido de autoayuda para el control de las emociones. Es decir, expresan conscientemente la relación bidireccional que actualmente se investiga entre el autocontrol y la AF. Como ejemplo, Finne et al. (2019) demostraron que el autocontrol promueve la actuación de las intenciones de hacer ejercicio a corto plazo. En el sentido opuesto, al considerar la autorregulación emocional como parte del autocontrol, se reconoce que la AF regular promueve el bienestar no solo al reducir los estados ansiosos de forma aguda, sino también al disminuir la inercia de ansiedad (Bernstein et al., 2019). Específicamente, Wienke y Jekauc (2016) describieron, desde un análisis cualitativo, los factores que promueven un estado emocional positivo en personas adheridas a la AF: la percepción de competencia, la interacción social, la experiencia novedosa y el esfuerzo físico.

En lo que concierne a la difusión de la $A F$, se encontró que hay una vía de influencia recibida desde los padres y las madres como también hay una necesidad de transmisión hacia la descendencia. Esto demuestra que la relación paterna/materna-filial es básica en la instauración de la motivación y del hábito del ejercicio. Un estudio demostró que los padres y las madres ejercen mayor influencia que el equipo docente de educación física en las prácticas deportivas de sus hijos e hijas (Olivares et al., 2015). Además, está documentado que, cuanto más crean los padres y las madres que las habilidades físicas mejoran con la práctica, más importante será para estos que sus descendientes participen en ella (Moreno-Murcia et al., 2018). 
Adicionalmente, cabe aclarar que no se detectaron indicadores psicológicos de sobreentrenamiento. Consideramos que esto obedece a los tipos de motivos señalados, los cuales son ajenos al rendimiento, en cuya área se reporta más este tipo de problemática (Sánchez-Beleña \& García-Naveira, 2017). Respecto a la adicción al EF, cuestión que en la actualidad tiende al aumento (Nogueira et al., 2017), no es posible determinar con claridad el alcance que tendrían los beneficios para la salud mental (como la reducción de estrés), descritos por la población participante, para asegurar que esté exenta de este padecimiento. Aunque se sabe que el riesgo de adicción al ejercicio se relaciona con peor calidad de vida y mayores trastornos alimenticios (Simón-Grima et al., 2019), estos indicadores no se encontraron en los entrevistados. Por otra parte, la autonomía fue uno de los aspectos destacados en los resultados. De acuerdo con Alcaraz-lbañez et al. (2020), la satisfacción de la necesidad de autonomía predice negativamente la adicción al ejercicio. En suma, no hay elementos puntuales que indiquen que en la muestra se presentó esta patología; sin embargo, tampoco puede descartarse ya que no se realizaron interrogantes directamente focalizadas en la conducta adictiva.

Es necesario advertir como una limitante del estudio el reducido tamaño de la muestra. Con mayor número de participantes, futuros estudios podrían profundizar sobre la experiencia de la vida activa diferenciada entre quienes han sido practicantes de algún deporte en equipo o de EF individual.

Otra limitación de este estudio es que todos los entrevistados han mantenido un peso corporal normal desde la infancia. Por tanto, no es generalizable a personas que se han adherido a la AF luego de un programa de reducción de peso o por alguna otra indicación médica. No obstante, una fortaleza del estudio radica en que se eligieron personas de una amplia gama de prácticas físico-deportivas y no solo de un centro deportivo o universitario.

Respecto a la aplicabilidad de los hallazgos a nivel de atención individualizada, para quienes planteen como motivo de consulta el deseo de crear un estilo de vida activo, se recomienda intervenir en la mejora de habilidades de autocontrol a fin de favorecer y acortar la brecha entre la intención y la conducta. Esto se puede dar a través de planes creados específicamente para la rutina del practicante. De igual manera, es relevante una valoración de los objetivos personales para posteriormente hacer una integración de un conjunto de motivos que permitan sostener la práctica a manera de red, aun cuando algunos no reciban retroalimentación inmediata. Asimismo, sería importante hacer explícitos los auto-diálogos de las personas para aumentar la conciencia respecto a su autocontrol y orientarlos hacia la consecución de la meta.

A un nivel de intervención social, se sugiere la aplicación de estos hallazgos en la planificación de publicidad dirigida a aumentar la adherencia a la AF:

1. Incorporar mensajes cortos con ejemplos del auto-diálogo motivante.

2. Presentar personajes con un perfil como el aquí descrito a fin de favorecer la identificación de personas que laboran y cuentan con poco tiempo libre, limitaciones económicas e hijos e hijas con un estilo de organización diaria que tenga a la AF como un hábito para mostrar cómo estas resuelven a su paso las barreras que se les presentan.

3. Resaltar los motivos de placer y de salud mental que mueven más a la permanencia en la actividad. 
De esta manera, se capitaliza la necesidad de difusión y se subraya la importancia que tiene la familia en la implantación del gusto por la AF, futuras investigaciones podrían diseñar una colaboración entre padres y madres de familia activos con cuerpos estudiantiles de escuelas de nivel básico.

En estudios subsecuentes, se podrían profundizar las características del auto diálogo para la auto-motivación, e incluso se podrían realizar comparaciones con personas que han abandonado la actividad física para encontrar puntos críticos que generan distintas respuestas conductuales.

Además, es recomendable un estudio más focalizado en el proceso del desarrollo del hábito del EF, ya que este factor parece ser la clave para un mantenimiento prolongado en la actividad. Esto se suma también al esclarecimiento de cómo un conjunto de motivos mixtos (intrínsecos y extrínsecos) apoya esta permanencia, ya que tradicionalmente se ha visto que las intervenciones en AF atienden mayormente a la fase de iniciación y se inclinan por fomentar la motivación intrínseca.

Finalmente, se reflexiona sobre el papel del profesional en psicología del deporte y de la AF en el tema de la lucha contra la inactividad física, el sedentarismo, la obesidad y la diabetes. Se considera que su participación es importante desde el contacto en primera instancia con la persona usuaria de servicios de salud y a nivel de la persona colaboradora en políticas públicas dirigidas a la activación. Los avances en la investigación muestran la complejidad del fenómeno, el aumento progresivo de los índices de las consecuencias de la inactividad, y finalmente, muestra que la orientación para quienes desean iniciar un estilo de vida activo (o quienes son urgidos a hacerlo) actualmente está exclusivamente en manos de profesionales de la medicina o técnicos del entrenamiento físico con poco conocimiento en la comprensión de la conducta humana. Un abordaje sanitario que contemple la motivación es necesario y urgente para lograr un cambio.

\section{Referencias}

Aaltonen, S., Rottensteiner, M., Kaprio, J., \& Kujala, U. M. (2014). Motives for physical activity among active and inactive persons in their mid-30s. Scandinavian Journal of Medicine \& Science in Sports, 24(4), 727-735. https://dx.doi.org/10.1111/sms.12040

Alcaraz-lbañez, M., Sicilia, Á., \& Lirola, M. J. (2020). Ansiedad físico social y adicción al ejercicio: análisis del papel mediador de las necesidades psicológicas básicas. Revista de Psicología del Deporte, 29, 24-33. https://rpd-online.com/index.php/ rpd/issue/download/3/1

Alvarado, M., Murphy, M. M., \& Guell, C. (2015). Barriers and facilitators to physical activity amongst overweight and obese women in an Afro-Caribbean population: A qualitative study. International Journal of Behavioral Nutrition \& Physical Activity, 12(97), 1-12. https://dx.doi.org/10.1186/s12966-015-0258-5

Ashton, L. M., Hutchesson, M. J., Rollo, M. E., Morgan, P. J., Thompson, D. I., \& Collins, C. E. (2015). Young adult males' motivators and perceived barriers towards eating healthily and being active: A qualitative study. International Journal of Behavioral Nutrition \& Physical Activity, 12(93), 1-10. https://dx.doi.org/10.1186/s12966-015-0257-6

Ball, J. W., Bice, M. R., \& Parry, T. (2014). Adults' motivation for physical activity: Differentiating motives for exercise, sport, and recreation. Recreational Sports Journal, 38(2), 130142. https://dx.doi.org/10.1123/rsj.2014-0048 
Bernstein, E. E., Curtiss, J. E., Wu, G. W. Y., Barreira, P. J., \& McNally, R. J. (2019). Exercise and emotion dynamics: An experience sampling study. Emotion, 19(4), 637-644. https://dx.doi.org/10.1037/emo0000462

Creswell, J. W. (2009). Research Design. Qualitative, Quantitative and Mixed Methods Approaches ( $3^{\mathrm{a}}$ ed.). Sage Publications.

Deci, E. L., \& Ryan, R. M. (2008). Self-determination theory: A macrotheory of human motivation, development, and health. Canadian Psychology/Psychologie canadienne, 49(3), 182-185. https://dx.doi.org/10.1037/a0012801

Finne, E., Englert, C., \& Jekauc, D. (2019). On the importance of self-control strength for regular physical activity. Psychology of Sport and Exercise, 43, 165-171. https:// dx.doi.org/10.1016/j.psychsport.2019.02.007

Gardner, B. (2015). A review and analysis of the use of 'habit' in understanding, predicting, and influencing health-related behavior. Health Psychology Review, 9(3), 277-295. https://dx.doi.org/10.1080/17437199.2013.876238

Gardner, B., Rebar, A. L., \& Lally, P. (2019). A matter of habit: Recognizing the multiple roles of habit in health behavior. British Journal of Health Psychology, 24(2), 241-249. https://dx.doi.org/10.1111/bjhp.12369

Goodsell, T., Harris, B., \& Bailey, B. (2013). Family status and motivations to run: A qualitative study of marathon runners. Leisure Sciences, 35(4), 337-352. https://dx.doi.org/10. 1080/01490400.2013.797326

Guillén, F., Castro, J. J., \& Guillén, M. A. (1997). Calidad de vida, salud y ejercicio físico: Una aproximación al tema desde una perspectiva psicosocial. Revista Psicología del Deporte, 12, 91-110. https://archives.rpd-online.com/article/view/461/451.html

Guillén, F., Weis, G., \& Navarro, M. (2005). Motivos de participación deportiva de niños atendiendo a sus edades. Apunts, 80(2), 29-36. https://revista-apunts.com/motivosde-participacion-deportiva-de-ninos-brasilenos-atendiendo-a-sus-edades/

Hartmann, C., Dohle, S., \& Siegrist, M. (2015). A self-determination theory approach to adults' healthy body weight motivation: A longitudinal study focusing on food choices and recreational physical activity. Psychology \& Health, 30(8), 924-948. https://dx.doi.org/10.1080/08870446.2015.1006223

Hennink, M. M., Kaiser, B. N., \& Marconi, V. C. (2017). Code saturation versus meaning saturation: How many interviews are enough? Qualitative Health Research, 27(4), 591-608. https://dx.doi.org/10.1177/1049732316665344

Instituto Nacional de Estadística y Geografía. (2018, 26 de enero). Menos de la mitad de la población realiza en su tiempo libre la práctica de algún deporte o ejercicio físico (Comunicado de prensa No. 25/18) [Comunicado de prensa]. https://www.inegi. org.mx/contenidos/saladeprensa/boletines/2018/mopradef/mopradef2018_01.pdf

Instituto Nacional de Salud Pública. (2016). Encuesta Nacional de Salud y Nutrición de Medio Camino 2016. https://www.gob.mx/cms/uploads/attachment/ file/209093/ENSANUT.pdf

Latinjak, A. T., Ramis, Y., Hatzigeorgiadis, A., \& Torregrossa, M. (2018). Autohabla y deporte: Una revisión interpretativa. Revista de Psicología del Deporte, 27(2), 75-86. https:// 
archives.rpd-online.com/article/view/v27-n2-latinjak-ramis-hatzigeorgiadis-etal/ Latinjak_Ramis_Hatzigeorgiadisetal.html

Lee, Y., \& Yoon, Y. J. (2019). Exploring the formation of exercise habits with the latent growth model. Perceptual \& Motor Skills, 126(5), 843-861. https://dx.doi. org/10.1177\%2F0031512519862689

Levesque, A., \& Li, H. Z. (2014). The relationship between culture, health conceptions and health practices: A qualitative-quantitative approach. Journal of Cross Cultural Psychology, 45(4), 628-645. https://dx.doi.org/10.1177/0022022113519855

Levitt, H. M. (2020). Reporting Qualitative research in Psychology: How to meet APA Style Journal Article Reporting Standards. American Psychological Association. https:// dx.doi.org/10.1037/0000179-000

Leyton, M., García-Matador, J., Fuentes, J. P., \& Jiménez, R. (2018). Análisis de variables motivacionales y de estilos de vida saludables en practicantes de ejercicio físico en centros deportivos en función del género. Retos: Nuevas Perspectivas de Educación Física, Deporte y Recreación, (34), 166-171. https://recyt.fecyt.es/index. php/retos/article/view/58281

López-Amador, K., \& Ocampo-Barrio, P. (2007). Creencias sobre su enfermedad, hábitos de alimentación, actividad física y tratamiento en un grupo de diabéticos mexicanos. Archivos en Medicina Familiar, 9(2), 80-86. http://www.medigraphic. com/pdfs/medfam/amf-2007/amf072c.pdf

Lozada-Tequeanes, A. L., Campero-Cuenca, M. L. E., Hernández, B., Rubalcava-Peñafiel, L., \& Neufeld, L. M. (2015). Barreras y facilitadores para actividad física durante el embarazo y posparto en mujeres pobres de México. Salud Pública de México, 57(3), 242-251. https://www.medigraphic.com/cgi-bin/new/resumen. cgi?IDARTICULO=58741

Migliorini, L., Cardinali, P., \& Rania, N. (2019). How could self-determination theory be useful for facing health innovation challenges? Frontiers in Psychology, 10(1870), 1-10. https://dx.doi.org/10.3389/fpsyg.2019.01870

Moreno-Murcia, J. A., Gea-Escámez, A., \& Corbí, M. (2018). Predicción de la percepción de actividad física de los hijos a partir de la creencia de habilidad de los padres. Cuadernos de Psicología del Deporte, 18(3), 1-11. https://revistas.um.es/cpd/ article/view/294291

Moreno-Murcia, J. A., Marcos-Pardo, P. J., \& Huéscar, E. (2016). Motivos de práctica físico-deportiva en mujeres: Diferencias entre practicantes y no practicantes. Revista de Psicología del Deporte, 25(1), 35-41. https://www.redalyc.org/ pdf/2351/235143645005.pdf

Ng, J. Y., Ntoumanis, N., Thøgersen-Ntoumani, C., Deci, E. L., Ryan, R. M., Duda, J. L., \& Williams, G. C. (2012). Self-determination theory applied to health contexts: A meta-analysis. Perspectives on Psychological Science, 7(4), 325-340. https:// dx.doi.org/10.1177/1745691612447309

Nogueira, A., Salguero del Valle, A., \& Márquez, S. (2017). Adicción a correr: Una revisión desde sus inicios hasta la actualidad. Revista de Psicología Aplicada al Deporte y al Ejercicio Físico, 1(6), 1-22. https://www.revistapsicologiaaplicadadeporteyejercicio. org/articulo.php?id=1f0e3dad99908345f7439f8ffabdffc4 
Ntoumanis, N., Healy, L. C., Sedikides, C., Duda, J., Stewart, B., Smith, A., \& Bond, J. (2014). When the going gets tough: The "why" of goal striving matters. Journal of Personality, 82(3), 225-236. https://dx.doi.org/10.1111/jopy.12047

Olivares, P. R., Cossio-Bolaños, M. A., Gómez-Campos, R., Almonacid-Fierro, A., \& GarcíaRubio, J. (2015). Influence of parents and physical education teachers in adolescent physical activity. International Journal of Clinical and Health Psychology, 15(2), 113-120. https://dx.doi.org/10.1016/j.ijchp.2015.01.002

Organización Mundial de la Salud. (2014). Informe sobre la situación mundial de las enfermedades no transmisibles 2014. http://apps.who.int/iris/ bitstream/10665/149296/1/WHO_NMH_NVI_15.1_spa.pdf?ua=1

Rodrigues, F., Bento, T., Cid, L., Pereira, H., Teixeira, D., Moutão, J., Almeida, D., \& Monteiro, D. (2018). Can interpersonal behavior influence the persistence and adherence to physical exercise practice in adults? A systematic review. Frontiers in Psychology, 9(2141), 1-15. https://dx.doi.org/10.3389/fpsyg.2018.02141

Rodríguez-Martínez, P., \& Guillen, F. (2017). Motivos de participación deportiva en jóvenes deportistas mexicanos. Cuadernos de Psicología del Deporte, 17(3), 105-116. https://revistas.um.es/cpd/article/view/313881

Rodríguez-Romo, G., Macías-Pla, R., Garrido-Muñoz, M., Tejero-González, C. M., \& LópezAdán, E. (2018). Motivos para la práctica de actividad física durante el tiempo libre y su relación con el cumplimento de las recomendaciones. Cuadernos de Psicología del Deporte, 18(1), 183-194. https://revistas.um.es/cpd/article/view/274141

Roychowdhury, D. (2018). Functional significance of participation motivation on physical activity involvement. Psychological Thought, 11(1), 9-17. https://dx.doi. org/10.5964/psyct.v11i1.255

Ruiz-Juan, F., Baena-Extremera, A., \& Baños, R. (2017). Nivel de actividad deportiva en el tiempo libre desde las etapas de cambio y motivación en estudiantes de Costa Rica, México y España. Cuadernos de Psicología del Deporte, 17(2), 53-64. https:// revistas.um.es/cpd/article/view/301891

Ryan, R. M., \& Deci, E. L. (2000). Self-determination theory and the facilitation of intrinsic motivation, social development, and well-being. American Psychologist, 55(1), 6878. https://dx.doi.org/10.1037/0003-066X.55.1.68

Ryan, R. M., Deci, E. L., Vansteenkiste, M., \& Soenens, B. (2021). Building a science of motivated persons: Self-determination theory's empirical approach to human experience and the regulation of behavior. Motivation Science, 7(2), 97-110. https://dx.doi.org/10.1037/mot0000194

Sánchez-Beleña, F., \& García-Naveira, A. (2017). Sobreentrenamiento y deporte desde una perspectiva psicológica: Estado de la cuestión. Revista de Psicología Aplicada al Deporte y al Ejercicio Físico, 2(12), 1-12. https://dx.doi.org/10.5093/rpadef2017a8

Sansone, C., \& Tang, Y. (2021). Intrinsic and extrinsic motivation and self-determination theory. Motivation Science, 7(2), 113-114. https://dx.doi.org/10.1037/mot0000234

Sarol, H., \& Çimen, Z. (2017). Why people participate leisure time physical activity: A turkish perspective. Pamukkale Journal of Sport Sciences, 8(1), 63-72. https://dergipark. org.tr/en/pub/psbd/issue/34046/615255 
Sebire, S. J., Standage, M., \& Vansteenkiste, M. (2008). Development and validation of the goal content for exercise questionnaire. Journal of Sport \& Exercise Psychology, 30(4), 353-377. https://dx.doi.org/10.1123/jsep.30.4.353

Secretaría de Salud. (2016, 14 de noviembre). Emite la Secretaría de salud emergencia epidemiológica por diabetes mellitus y obesidad [Comunicado de prensa]. https://www.gob.mx/salud/prensa/emite-la-secretaria-de-salud-emergenciaepidemiologica-por-diabetes-mellitus-y-obesidad

Sicilia, Á., González-Cutre, D., Artés, E. M., Orta, A., Casimiro, A. J., \& Ferriz, R. (2014). Motivos de los ciudadanos para realizar ejercicio físico: Un estudio desde la teoría de la autodeterminación. Revista Latinoamericana de Psicología, 46(2), 8391. https://dx.doi.org/10.1016/S0120-0534(14)70011-1

Simón-Grima, J., Estrada-Marcén, N., \& Montero-Marín, J. (2019). Adicción al ejercicio medida a través del Exercise Addiction Inventory (EAI) y salud en deportistas habituales. Una revisión sistemática y meta-análisis. Adicciones, 37(3), 233-249. https://doi.org/10.20882/adicciones.990

Smith, B., \& McGannon, K. R. (2018). Developing rigor in qualitative research: Problems and opportunities within sport and exercise psychology. International Review of Sport and Exercise Psychology, 11(1), 101-121. https://dx.doi.org/10.1080/1750984X.2017.1317357

Stelzer, J., Klimesova, I., Ruzicka, M., \& Ernest, J. (2018). The Connection between motivation and behaviors. En J. Vopava, V. Douda, R. Kratochvil, \& M. Konecki (Eds.), Proceedings of the Multidisciplinary Academic Conference (pp. 182-189). Academic Conferences Association. https://rke.abertay.ac.uk/ws/portalfiles/portal/15089829/ ProceedingsOfMAC2018InPrague_Published_2018.pdf\#page=188

Teixeira, P. J., Carraça, E. V., Markland, D., Silva, M. N., \& Ryan, R. M. (2012). Exercise, physical activity, and self-determination theory: A systematic review. International Journal of Behavioral Nutrition and Physical Activity, 9(78), 1-30. https://dx.doi. org/10.1186/1479-5868-9-78

Valenzuela, R., Codina, N., \& Pestana, J. V. (2021). University students' motives-for-physicalactivity profiles: Why they practice and what they get in terms of psychological need satisfaction. Frontiers in Psychology, 11(621065). https://dx.doi.org/10.3389/ fpsyg.2020.621065

Wienke, B., \& Jekauc, D. (2016). A qualitative analysis of emotional facilitators in exercise. Frontiers in Psychology, 7(1296). https://dx.doi.org/10.3389/fpsyg.2016.01296

World Medical Association. (2013). World Medical Association Declaration of Helsinki: Ethical principles for medical research involving human subjects. JAMA, 310(20), 2191-2194. https://dx.doi.org/10.1001/jama.2013.281053 